\title{
When right is left and left is right: using the JL3.5 guide catheter for left and right coronary PCI
}

\author{
Vaidya $\mathrm{K}^{1}$ and Lowe $\mathrm{HC}^{1,2 *}$ \\ ${ }^{1}$ Cardiology Department, Orange Base Hospital, Orange, Australia \\ ${ }^{2}$ Cardiology Department, Concord Repatriation General Hospital, Concord, Australia
}

\begin{abstract}
A case is presented using the Judkins Left (JL 3.5) guide catheter to cannulate both the left main coronary artery and then the right coronary artery for multivessel transradial coronary intervention, to highlight this straightforward, novel approach.
\end{abstract}

\section{Introduction}

Transradial-approach coronary angiography and percutaneous coronary intervention (PCI) has minimised rates of access site complications but has required a re-evaluation of guide catheter selection to optimise procedural success [1,2]. A shortcoming of transradial intervention may often be poor backup support, especially for the Right Coronary Artery (RCA), due to the angle between the subclavian and brachiocephalic arteries which may prevent optimal positioning of the catheter along the contralateral aortic wall [3]. A variety of radial-dedicated guide catheters have been proposed to overcome these challenges, including the Kimney, Fadajet, Barbeau, and Ikari Left catheters. The Ikari Left in particular - essentially a modified Judkin's catheter - claims better backup support, deeper engagement, and has been proposed as a "single-catheter" for both the left and right coronary artery (1). However, these catheters have not been widely adopted, with contemporary practice still favouring traditional guide catheters: (standard extra backup (EBU/XB; 77-81\%) and Judkins Left (9-17\%) for the left coronary, and Judkin's Right (JR; 74-81\%) and Amplatz Left (AL; 8-10\%) for the right coronary, in recent surveys $[2,4]$.

\section{Case report}

The use of a JL3.5 as a dual-purpose guide catheter for a sequential left and right transradial intervention was performed in a 62 year old male presenting with a non ST segment elevation myocardial infarction, with an LAD lesion and then an RCA lesion being treated sequentially using the same JL3.5 guide catheter (Figure 1). Importantly, a standard 0.035 " wire was used to extend the greater curve of the guide prior to RCA cannulation (Figure 1), in an uncomplicated procedure with excellent guide support, as has been previously described [5]. Despite discrepancies of shape without a support wire, the JL catheter bears a strong resemblance to the transradial diagnostic Tiger (TIG) catheter, which cannulates both the LCA and RCA, when the secondary curve is straightened with a guidewire (Figure 2). It has been suggested that the backup support of a JL3.5 for the RCA is as robust as most other techniques [5].
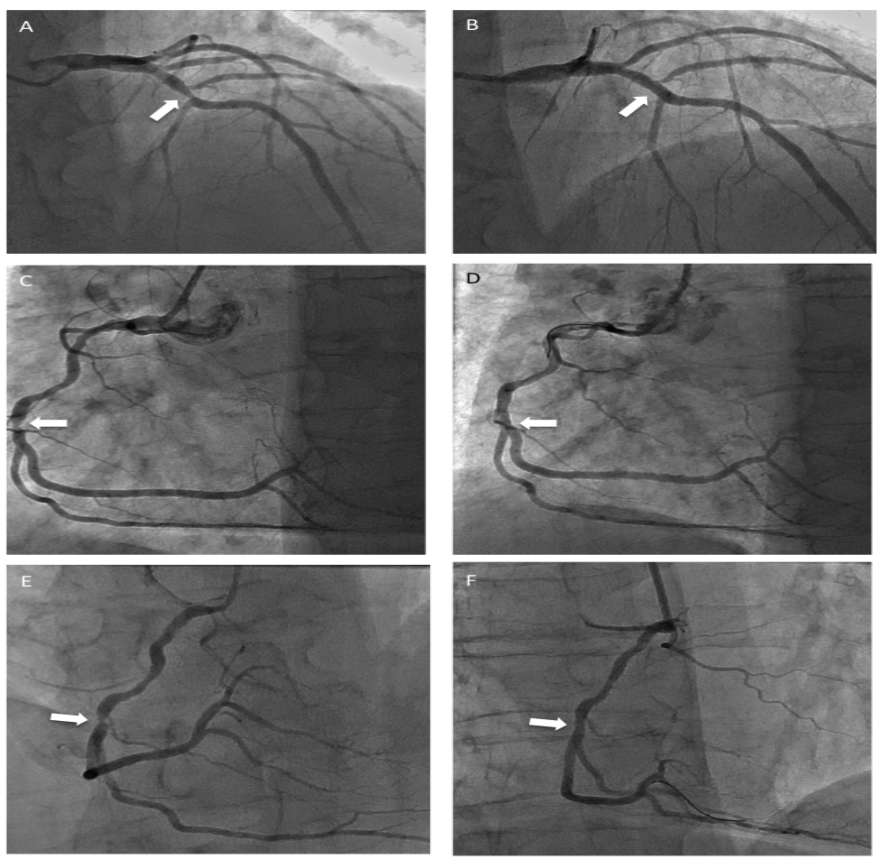

Figure 1. Coronary Intervention Using a 6F JL3.5 guide via the right radial approach

A,B. Left Anterior Descending lesion (RAO Cranial view). The lesion (white arrow is shown pre (A) and post (B) stent, with JL3.5 as guide catheter

C,D. Right Coronary Artery lesion (white arrow) in LAO view pre (C) and post (D) stent, using same JL 3.5 guide catheter

E, F. Right Coronary Artery lesion (white arrow) in RAO view pre (E) and post (F) stent, using same JL 3.5 guide catheter

${ }^{\star}$ Correspondence to: Lowe HC, Associate Professor, Cardiology Department, Concord Repatriation General Hospital, Concord, NSW, 2139, Australia, Tel: 6129767 5000, E-mail: h.lowe@bigpond.net.au

Key words: guide catheter, stent

Received: May 04, 2020; Accepted: May 18, 2020; Published: May 25, 2020 

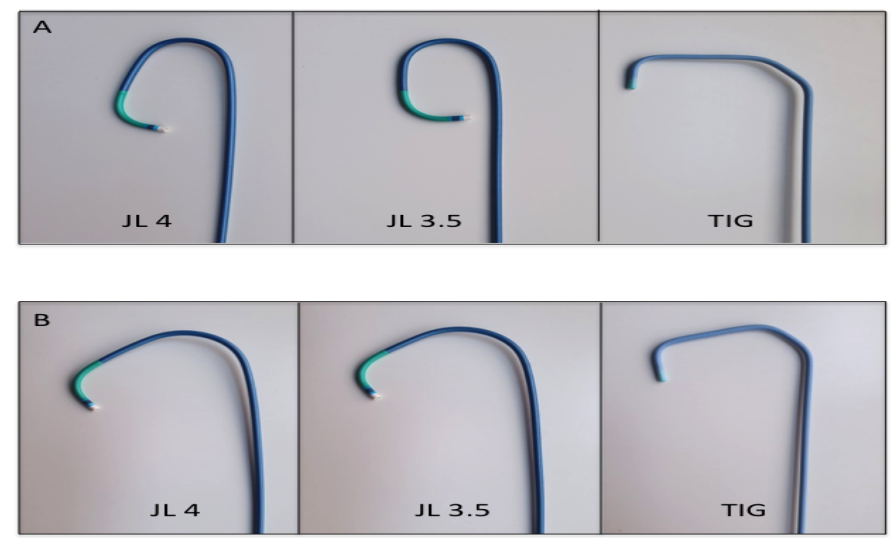

Figure 2. Comparison of shapes of 6F JL4, JL3.5 guide catheters and TIG 6F diagnostic catheters

A. Catheter shapes without 0.035 " metal introducer wire in situ: The JL4 and JL3.5 preserve an acute angle of the greater curve

B. Catheter shapes with 0.035 " metal introducer wire in situ: The JL4 and JL3.5 open the greater curve, with the TIG virtually unchanged, rendering the JL3.5 in particular almost analogous to the TIG in curve shape, suggesting feasibility of right coronary cannulation

\section{Conclusion}

The technique used here is not novel [5] but does not feature in recent surveys of guide catheter use [2,4]. We would therefore suggest this readily available "single use guide catheter" - along with the technique of RCA cannulation with wire-assisted greater curve extension - is further examined.

\section{References}

1. Ponangi U (2014) Current and emerging catheter technologies for percutaneous transluminal coronary angioplasty. Res rep clin cardiol 5: 213-226.

2. Bertrand OF, Rao SV, Pancholy S, Jolly SS, Rodes-Cabau J, et al. (2010) Transradial approach for coronary angiography and interventions: results of the first international transradial practice survey. JACC Cardiovasc Interv 3: 1022-1031. [Crossref]

3. Ikari Y, Masuda N, Matsukage T, Ogata N, Nakazawa G, et al. (2009) Backup force of guiding catheters for the right coronary artery in transfemoral and transradial interventions. J Invasive Cardiol 21: 570-574. [Crossref]

4. Shroff AR, Fernandez C, Vidovich MI, Rao SV, Cowley M, et al. (2019) Contemporary transradial access practices: Results of the second international survey. Catheter Cardiovasc Interv 93: 1276-1287. [Crossref]

5. Kaul S, Kaul N (2017) JL 3.5/4 as a multipurpose guiding catheter for simultaneous multivessel (left and right) transradial PCI. IHJ Cardiovasc Case Rep (CVCR) 1: 75-76.

Copyright: $\odot 2020$ Vaidya K. This is an open-access article distributed under the terms of the Creative Commons Attribution License, which permits unrestricted use, distribution, and reproduction in any medium, provided the original author and source are credited. 